\title{
APRENDIZAGEM BASEADA EM PROJETOS APLICADA AO ENSINO DE ENGENHARIA: MOTIVAÇÃO E MOBILIZAÇÃO DE COMPETÊNCIAS
}

Francisco Santos Sabbadini-franciscosabbadini@gmail.com

Universidade do Estado do Rio de Janeiro, Departamento de engenharia de produção Projeto Inovativas

Rodovia Presidente Dutra km 298

27500-000 - Resende - RJ

Wesley Messias Mendonça Faria -wesleymmf@hotmail.com

Universidade do Estado do Rio de Janeiro, Departamento de engenharia de produção

Projeto Inovativas

Rodovia Presidente Dutra km 298

27500-000-Resende - RJ

Reinaldo de Oliveira Silva-e-mail*

Universidade do Estado do Rio de Janeiro, Departamento de engenharia de produção

Projeto Inovativas

Rodovia Presidente Dutra km 298

27500-000 - Resende - RJ

Resumo: A nova relação entre tecnologia, produção e emprego trouxe um desafio às instituições de ensino, com relação a formação de profissionais no mundo todo. O caminho apontado é do ensino voltado para as competências, como uma resposta a esse desafio, uma vez que o modelo atual baseado no método tradicional não se mostra efetivo. Isso pode estar relacionado ao fato de que sua abordagem conservadora e passiva a torna ineficiente $e$ desmotivadora para os estudantes. As metodologias ativas e, entre elas, a Aprendizagem Baseada em Projetos, estão alinhadas com as demandas dos cursos de Engenharia em face da nova realidade contemporânea. No presente artigo se apresentam os resultados da busca para compreender, por meio de um questionário de avaliação com escala simétrica, a percepção de estudantes de engenharia após a aplicação da Aprendizagem Baseada em Projetos, em uma disciplina do ciclo profissionalizante, durante um semestre letivo, com a participação de uma empresa do segmento automotivo. Os resultados indicaram a efetividade dessa metodologia com relação a mobilização das competências e da motivação dos alunos.

Palavras-chave: Aprendizagem Baseada em Projetos. Metodologias ativas. Ensino de Engenharia.

\section{INTRODUÇÃO}

O mundo, o trabalho e a sociedade como um todo tem sido impactados pelas novas tecnologias de informação e comunicação, que tornaram as interações sociais mais intensivas, assim como a velocidade na produção de conhecimento. Junto a isso, as tecnologias de automação, análise de dados, inteligência artificial, impressão 3D (manufatura aditiva), base da 
indústria 4.0 tem gerado preocupação com o tipo de profissional que será requerido para o século 21 e depois.

Na pesquisa sobre desenvolvimento mundial, que trata relação entre tecnologia, produção e emprego, o Banco Mundial (2019), constatou que muitos jovens em formação hoje irão trabalhar no futuro com tecnologias e em profissões que ainda não existem. Neste sentido, sugere que a superação da lacuna entre o aprendizado do presente e as necessidades da nova economia do futuro, baseada no conhecimento, se dará pelo desenvolvimento das competências socioemocionais dos profissionais do futuro.

Os currículos dos atuais cursos de engenharia no Brasil, estruturados de uma maneira estanque, com os conhecimentos compartimentalizados em disciplinas isoladas, não correspondem as demandas do mercado e da sociedade em relação ao profissional que se forma, uma vez que sua formação não comporta a multidisciplinaridade requerida para os profissionais nos dias atuais (MANRIQUE; DIRANI; CAMPOS, 2010). Num modelo ainda fortemente centrado no professor, estão baseados em sua maioria na lógica do conteúdo factual e não nas estratégias de aprendizagem mobilizadoras (FURTADO, 2013), e consequentemente não orientados para o ensino centrado no aluno e para o desenvolvimento das suas competências.

Para promover e estimular as ações de modo que a formação dos futuros engenheiros esteja alinhada com as demandas sociais e do trabalho, a resolução nr. 2 de 24 de abril de 2019, instituiu as Diretrizes Curriculares Nacionais (DCN) dos cursos de engenharia, considerando no seu parágrafo 6 , o estimulo para o uso das metodologias de aprendizagem ativa e no parágrafo 7, o ensino centrado no aluno (MEC, 2019).

No sentido de contribuir para o ensino da engenharia, no âmbito da aprendizagem ativa, este artigo apresenta os resultados de uma prática implementada em uma universidade pública brasileira, por meio de uma abordagem pedagógico-metodológica de aprendizagem baseada em projetos.

\section{FUNDAMENTAÇÃO}

Nesta seção serão apresentados os fundamentos conceituais que serviram de base para o presente estudo, considerando a questão da aprendizagem ativa, das competências e da Aprendizagem Baseada em Projetos.

\subsection{Aprendizagem ativa e metodologias}

O aprendizado ativo segundo Aknoglu e Tandogan (2007) é um processo centrado no aluno no qual este trás para si a responsabilidade por sua aprendizagem e recebe a oportunidade de tomar decisões sobre várias dimensões contextuais com autonomia. Nesta abordagem o professor deixa de ser o centro do processo, sendo o gestor das estratégias de ensino.

De acordo com Camargo e Daros (2018) o aprendizado ativo propicia ao estudante desenvolver seu protagonismo, por meio do trabalho colaborativo e na interação como outros alunos. As metodologias ativas se aplicam como alternativa ao modelo tradicional, uma vez que são orientadas para formas de desenvolver o processo de aprender, baseado em experiências reais, com vistas a resolver desafios e problemas de ordem social ou profissional em diferentes contextos. Ainda, de acordo com esses autores, motiva o aluno pois ele consegue atribuir significado ao que está estudando e aprendendo.

No Brasil, algumas instituições de ensino tem procurado desenvolver ações nesse sentido, em termos de práticas de ensino-aprendizagem e organização currícular, visando desenvolver nos alunos a motivação e a autonomia no aprender. Cabe destaque a criação do consórcio formado entre Instituições de Ensino Superior (IES) brasileiras e o LASPAU, organismo filiado 
à Universidade de Harvard University (EUA) dedicado disseminar inserção dessas metodologias ativas em sala de aula (SEMESP, 2014), dentre as quais são destacadas: Aprendizagem baseada em problemas (PBL), Aprendizagem Baseada em Projetos, Peer Instruction (MAZUR, 2015), Just-in-time Teaching (JitT), Aprendizagem baseada em times (TBL), métodos de caso e simulações. Uma explicação detalhada pode ser vista em Rocha e Lemos (2014). A aprendizagem baseada em projetos foi a metodologia ativa utilizada no presente estudo.

\subsection{Aprendizagem baseada em projetos}

Essa abordagem metodológica é um modelo de ensino que consiste essencialmente em permitir que os estudantes possam confrontar questões do mundo real que sejam significativas e definir uma forma estruturada de trabalhar sobre o mesmo, de modo cooperativo em busca de soluções (BENDER, 2014), permitindo aos alunos uma investigação com base nos conceitos e teorias da ou das disciplinas que estejam relacionadas. Neste sentido é um método integrador de conhecimentos.

Outro aspecto a ser considerado é o de que além de possibilitar o aprendizado, esta metodologia, segundo Cole, Wasburn-Moses (2010) aumenta a motivação para aprender e trabalhar em equipe. Ainda segundo esses autores contribui para desenvolver habilidades colaborativas. Segundo Larmer e Mergen-Doller (2010) é uma abordagem que resulta em níveis elevados de envolvimento e desempenho dos alunos.

Do ponto de vista da engenharia, os benefícios desta Aprendizagem Baseada em Projetos, apontados por Rocha e Lemos (2014), estão no enquadramento dos problemas nos contextos culturais e sociais, e na necessidade de adaptação do aluno no processo de aprendizagem à medida que os problemas se tornam imprevisíveis na sala de aula, como ocorre na vida profissional.

Nesse sentido diferentemente do método tradicional de ensino, com aulas expositivas centrado no domínio do professor, experiências de aprendizagem ativa contribuem para o desenvolvimento das competências múltiplas, entre as quais as socioemocionais.

\subsection{Competências}

A introdução das competências nas diretrizes curriculares do ensino superior, vieram ao encontro das demandas atuais do mercado e da sociedade. Nesse sentido competência pode ser entendida no sentido latu como a mobilização de conhecimentos, habilidades, atitudes e valores, para o enfrentamento dos desafios do cotidiano e do mundo do trabalho, incluindo-se os valores dos indivíduos.

No presente estudo o entendimento de competência é o mesmo apontado por Silva (2007) "como a demonstração, dentro de situações concretas, de domínio de saberes e de habilidades, apreendidos e aprendidos, promovendo a capacidade de inovar com autonomia nas decisões e ações", devidamente ajustado ao contexto do ensino de engenharia. E do mesmo modo incorpora elementos de 03 dimensões: cognitiva, não cognitiva e comportamental (UNESCO, 2015), conforme mostra o "Quadro 1". 
Quadro 1 - Dimensões e competências relacionadas no estudo

\begin{tabular}{|c|c|c|}
\hline Dimensão & Conceito & Competência \\
\hline Cognitiva & $\begin{array}{l}\text { Pensar de forma crítica, sistêmica e criativa, } \\
\text { incluindo a adoção de uma abordagem de múltiplas } \\
\text { perspectivas, que reconheça diferentes dimensões, } \\
\text { perspectivas e ângulos de questões (como } \\
\text { habilidades de raciocínio e de resoluções de } \\
\text { problemas, apoiadas por uma abordagem de } \\
\text { múltiplas perspectivas) }\end{array}$ & $\begin{array}{l}\text { Criatividade } \\
\text { Pensamento crítico } \\
\text { Tomada de decisão }\end{array}$ \\
\hline Não cognitivas & $\begin{array}{l}\text { Habilidades sociais, como empatia e resolução de } \\
\text { conflitos, e habilidades de comunicação e aptidões } \\
\text { para networking e para a interação com pessoas de } \\
\text { diferentes contextos, origens, culturas e } \\
\text { perspectivas (como empatia global e sentimento de } \\
\text { solidariedade) }\end{array}$ & $\begin{array}{ll}\begin{array}{l}\text { Comunicação } \\
\text { Trabalho } \\
\text { equipe }\end{array} & \text { em } \\
\begin{array}{l}\text { Liderança } \\
\text { Resolução } \\
\text { conflitos }\end{array} & \\
\end{array}$ \\
\hline Comportamentais & $\begin{array}{l}\text { Agir de forma colaborativa e responsável, a fim de } \\
\text { encontrar soluções globais para desafios globais, } \\
\text { bem como para lutar pelo bem coletivo (como } \\
\text { sentimento de compromisso e habilidades de } \\
\text { tomadas de decisão) }\end{array}$ & Colaboração \\
\hline
\end{tabular}

Fonte: Elaborado com base em Unesco (2015).

As competências relacionadas no quadro 1, são as que foram investigadas nesta pesquisa e serão apresentadas nas discussões neste relato do estudo em andamento.

\section{METODOLOGIA}

A abordagem utilizada foi a da metodologia ativa de aprendizagem por projeto, aplicada numa disciplina do ciclo profissionalizante, relacionada aos fundamentos do gerenciamento da produção, nos cursos de Engenharia de Produção e Engenharia Mecânica, no ano de 2019.

Os projetos foram desenvolvidos com o apoio de uma empresa do setor automotivo da região Sul Fluminense, no estado do Rio de Janeiro, que viabilizou aos alunos o acesso às informações e processos produtivos, mediante a assinatura de termo de confidencialidade. Por razões instituicionais o nome da empresa não será citado neste artigo.

Por questões de segurança, devido ao alto risco de acidentes decorrentes da natureza da produção, os alunos não tiveram acesso físico aos locais e setores. Uma equipe da empresa se encarregou de filmar as áreas de produção, durante as atividades diárias, relativas à cada projeto.

Participaram 32 alunos, do quarto ao oitavo período, sendo 08 do curso de engenharia mecânica e 19 do curso de engenharia de produção. A turma foi dividida em 5 grupos, ficando cada grupo responsável por um projeto e com tutoria por um dos 5 profissionais da empresa, designados para apoiar cada equipe. 
O conteúdo conceitual abrangeu temas como: prioridades competitivas em gestão de operações, mapeamento e análise de processos, gestão da qualidade, melhoria da qualidade, gestão da capacidade, ergonomia, teoria das restrições, sistemas de produção, gestão de projetos, simulação e cronoanálise.

Para realização das atividades foram definidas as seguintes etapas:

1) Durante a primeira metade do semestre: aulas com método de ensino tradicional: aula expositiva. Arranjo tradicional da sala, com carteiras alinhadas e o professor na frente da sala. A cada duas semanas aplicação de exercícios de revisão.

2) $\mathrm{Na}$ segunda metade do semestre (08 aulas): Método de projetos. Sala de aula invertida, com o estudo antecipado uma semana antes, do conteúdo da aula seguinte. O período inicial da aula de 30 a 40 minutos reservado para o professor tirar dúvidas e fazer apresentações curtas de algum ponto que requeresse reforço.Arranjo da sala: por equipes. Após sanar dúvidas, cada equipe desenvolvia o seu projeto. Nesta fase da aula, os tutores da empresa participavam tirando dúvidas do projeto de suas respectivas equipes. A cada duas semanas, aplicação de exercícios de revisão.

3) Entrega do projeto: Mediante a apresentação de um seminário, contando com a presença além do professor e dos tutores, de gerentes dos setores aos quais se relacionavam os projetos e do diretor geral da empresa. Para essa fase, uma semana antes, cada equipe entregou um relatório escrito do projeto e fez uma apresentação prévia com a presença apenas do professor e tutores, para ajustes e correções.

4) Realização do seminário final.

A divisão das aulas em método tradicional e método de aprendizagem por projetos, teve como objetivo avaliar a percepção dos alunos quanto às duas formas de ensino. Ao final da etapa de cada abordagem metodológica foi investigado o seu grau de motivação com relação a metodologia de ensino utilizada naquela fase. A forma definida para isso foi por meio da coleta de respostas através de questionário, com respostas abertas e fechadas, o qual foi respondido por cada aluno participante da disciplina.

Para isso foi disponibilizado um formulário eletrônico desenvolvido no Google Forms. Antes de iniciar o preenchimento do questionário, os alunos foram informados que se tratava de uma pesquisa acadêmica, com a divulgação dos resultados por meio de um artigo científico, e de que suas respostas seriam confidenciais. Foi informado, ainda, aos estudantes que a resposta ao questionário seria expontânea e de que não estava vinculada a nenhuma atribuição de nota. Do mesmo modo foi solicitado que dessem sua concordância.

A avaliação dos resultados foi realizada por meio de uma escala de 1 a 10 , na qual os participantes responderam ao questionário atribuindo uma nota em uma escala simétrica para cada item, no sentido de que o resultado obtido indicasse a intensidade da sua percepção em relação ao tópico pesquisado. A fim de permitir uma compreensão integrada das percepções dos alunos, o questionário foi composto por 5 questões, segmentadas em 3 partes:

\section{Mobilização das competências}

a. Dê uma nota de 1 a 10 para cada uma das competências abaixo, considerando o grau de utilização da mesma por você para o desenvolvimento do projeto: colaboração, 
comunicação, criatividade, liderança, pensamento crítico, resolução de conflitos, tomada de decisão e trabalho em equipe.

2. Abordagem de ensino

a. Qual das abordagens de ensino você considera que mais contribuiu para o seu aprendizado:

( ) Tradicional

( ) Por projetos

b. Por que?

3. Motivação

a. Qual o seu grau de motivação na aula tradicional?

b. Qual o seu grau de motivação na aula por projetos?

\section{RESULTADOS E DISCUSSÃO}

Dos 32 alunos participantes, 27 responderam ao questionário, representando $84,37 \%$ do total. Desses, todos responderam integralmente a todas as questões propostas.

Para melhor compreensão dos resultados a seguir, a análise foi estruturada de acordo com as seguintes dimensões: mobilização das competências, abordagem de ensino e motivação. No primeiro foram analisadas as impressões dos alunos em relação ao quanto eles necessitaram utilizar as competências durante as aulas de Aprendizagem Baseada em Projetos. Os resultados são apresentados na "Tabela 1".

Tabela 1 - Percepção dos alunos no uso das competências

\begin{tabular}{l|c|c|c|c|c|c|c|c|c|c|c|}
\hline Competência & 1 & 2 & 3 & 4 & 5 & 6 & 7 & 8 & 9 & 10 & Total \\
\hline Colaboração & 0,000 & 0,000 & 0,000 & 0,000 & 0,000 & 0,000 & 0,000 & 0,074 & 0,259 & 0,667 & 1,000 \\
\hline Comunicação & 0,000 & 0,000 & 0,000 & 0,000 & 0,000 & 0,037 & 0,037 & 0,074 & 0,296 & 0,556 & 1,000 \\
\hline Criatividade & 0,000 & 0,000 & 0,000 & 0,000 & 0,000 & 0,111 & 0,111 & 0,185 & 0,259 & 0,333 & 1,000 \\
\hline Liderança & 0,000 & 0,000 & 0,000 & 0,037 & 0,037 & 0,074 & 0,074 & 0,074 & 0,296 & 0,407 & 1,000 \\
\hline Pensamento crítico & 0,000 & 0,000 & 0,000 & 0,000 & 0,000 & 0,000 & 0,000 & 0,222 & 0,333 & 0,444 & 1,000 \\
\hline Resolução de conflitos & 0,000 & 0,000 & 0,000 & 0,000 & 0,037 & 0,037 & 0,037 & 0,259 & 0,185 & 0,444 & 1,000 \\
\hline Tomada de decisão & 0,000 & 0,000 & 0,000 & 0,000 & 0,000 & 0,037 & 0,074 & 0,259 & 0,296 & 0,333 & 1,000 \\
\hline Trabalho em equipe & 0,000 & 0,000 & 0,000 & 0,000 & 0,000 & 0,037 & 0,148 & 0,074 & 0,148 & 0,593 & 1,000 \\
\hline
\end{tabular}

Fonte: Autores, 2019.

Os resultados sugerem que houve um grau significativo de envolvimento dos alunos. Todas as competências pesquisadas foram mobilizadas, sendo que colaboração e comunicação foram as mais utilizadas segundo a percepção dos estudantes. Quando consideradas as notas 9 e 10 para ambas, os escores são respectivamente, $0,926(92,6 \%)$ e $0,852(85,2 \%)$. O que sugere que com o desenvolvimento do projeto houve uma intensificação na colaboração mútua e na comunicação. Outro fator que pode ter contribuído para isso e ainda merece investigação é com relação a competência de resolução de conflitos, que apresentou um escore alto indicando que 
houve um considerável nível de tensão nas equipes, isso conforme observado durante o semestre, muito em decorrência das exigências de prazos de entrega dos projetos.

Das competências não cognitivas (comunicação, liderança, trabalho em equipe e resolução de conflitos), baseadas em habilidades sociais, a que mais chamou a atenção foi a liderança, que apresentou um grau de dispersão maior com as notas variando de 4 a 10 . Não foram identificados elementos na pesquisa que explicassem essa dispersão, embora a despeito disso tenha um resultado de alto escore de $0,704(70,4 \%)$ nos extremos 9 e 10 . Na mesma medida o trabalho em equipe apresentou um escore alto de 0,741 , o que parece confirmar as colocações de Cole, Wasburn-Moses (2010), de que a Aprendizagem Baseada em Projetos aumenta a motivação para trabalhar em equipe.

Em relação as competências cognitivas (criatividade, pensamento crítico e tomada de decisão) pesquisadas, os resultados mostraram que a mobilização da criatividade foi elevada na aprendizagem baseada em projetos, quando considerado os escores extremos 9 e 10. Em contrapartida com as demais, pensamento crítico e tomada de decisão embora tenham sido utilizadas, o foram em menor grau, com resultados de 0,593 e 0,630 para tomada de decisão, respectivamente.

$\mathrm{Na}$ segunda dimensão, foram analisadas as percepções dos estudantes em relação a abordagem de ensino baseada em projetos comparada com a visão dos mesmos sobre as metodologias tradicionais de ensino. O resultado é mostrado na "Figura 1".

Figura 1 - Contribuição para a aprendizagem.

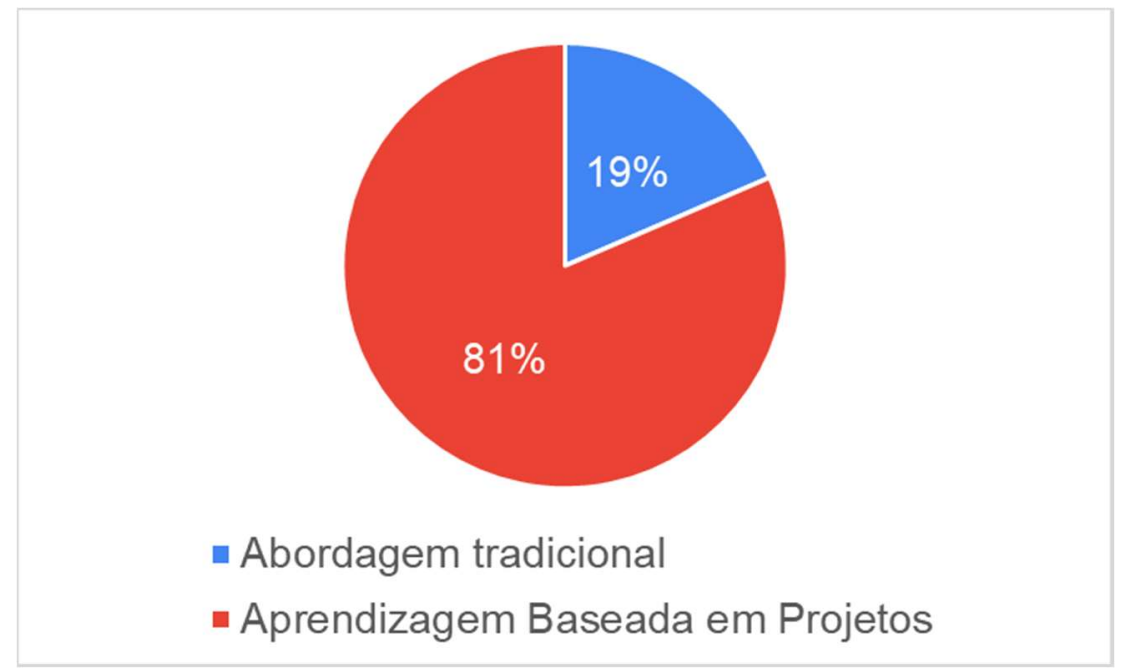

Fonte: Autores, 2019

A análise das razões elencadas pelos alunos em relação a sua percepção de contribuição para a aprendizagem, em relação a aprendizagem baseada em projetos, foram: 1) A possibilidade de colocar a teoria em prática $(51,85 \%)$; 2) Ter acesso a situações reais do que ocorre numa empresa $(25,93 \% ; 3)$ Estimular a criatividade $(11,1 \%)$; e 4) Outros motivos como melhorar a atitude, raciocínio e networking foram elencados. Em relação a abordagem tradicional, o principal argumento apresentado é a preferência por aulas com esse formato. 
Na terceira, a percepção da motivação dos mesmos comparativamente em relação ao ensino tradicional e o ensino por meio de projetos. Considerando a escala de graus de 1 a 10, a "Figura $2 "$ mostra os resultados.

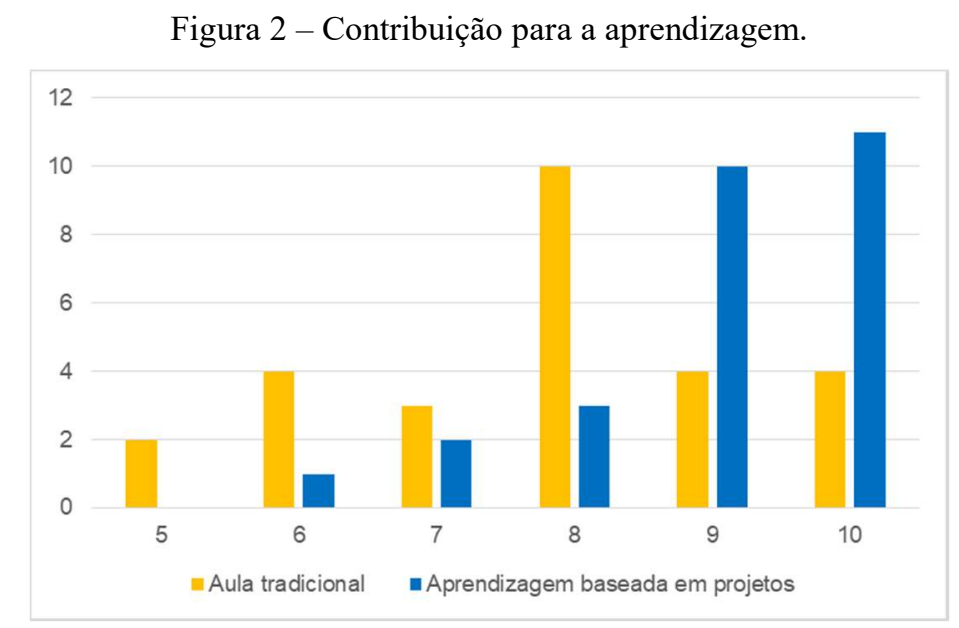

Fonte: Autor, 2019

Conforme mencionado na metodologia, o semestre letivo foi divido, sendo metade das aulas na metodologia tradicional e a outra metade com a metodologia de aprendizagem baseada em projetos. Ao final de cada ciclo os alunos responderam sobre sua motivação com relação aquele método de ensino. Na "Figura 2" se pode perceber que eles atribuíram grau 8 e alguns 9 e 10, sugerindo que estavam motivados com o método tradicional, com o qual já vinham convivendo ao longo da sua formação educacional. Ao se comparar com os resultados da aprendizagem baseada em projetos, o grau de motivação com essa metodologia é nas faixas de graus 8,9 e 10, sendo que nestas duas últimas notas superam em muito as avaliações em relação ao método tradicional, o que corrobora Cole, Wasburn-Moses (2010) quanto a motivação para aprender propiciada por aquela metodologia.

\section{CONSIDERAÇÕES FINAIS}

O presente estudo avaliou a percepção dos alunos quanto a utilização de competências, assim como da motivação dos mesmos em relação a abordagem tradicional de ensino e a aprendizagem baseada em projetos.

Os resultados da pesquisa, indicaram que a aprendizagem baseada em projetos apresenta maior grau de motivação no que diz respeito a aprendizagem e ao trabalho em equipe, na percepção dos alunos. Do mesmo modo sugerem que há uma maior empenho e envolvimento dos estudantes, por meio deste método, pela possibilidade efetiva de associarem a teoria com a prática.

No mesmo sentido, permitiram evidenciar a relevância do acesso dos estudantes ao ambiente empresarial e à realidade das operações de uma empresa, como é o caso deste estudo em que puderam ter contato efetivo com situações reais ligadas a produção no setor automotivo.

Oportunidades para estudos futuros estão na avaliação da correlação entre as variáveis cognitivas, não cognitivas e comportamentais, como por exemplo comunicação, colaboração e 
resolução de conflitos. Além disso, se verificou na pesquisa que 19\% dos alunos consideram que a metodologia tradicional de ensino contribui mais para seu aprendizado. É um percentual elevado cujas razões merecem ser investigadas, por meio de inquérito direcionado a aqueles respondentes. Por fim, a aplicação da presente pesquisa em outros disciplinas e cursos.

\section{Agradecimentos}

Nossos agradecimentos à direção da empresa do setor automotivo, sua equipe de gestores e tutores, que deram integral apoio antes, durante e depois do desenvolvimento deste estudo.

\section{REFERÊNCIAS}

AKINOGLU, O.; TANDOJAN, O. The effects of problem-based active learning in science education on students academic achieviment, attitude and concept learning. Eurasia Journal of Mathmatics, Science and Technology Education. 3 (1), pp. 305-315, 2015.71-81, 2007.

BANCO MUNDIAL. World Development Report 2019: the changing nature of work. Washington: The World Bank, 2019.

BENDER, Willian N. Aprendizagem baseada em projetos: educação diferenciada para o século XXI. 1'. Ed. Porto Alegre: Penso, 2014.

CAMARGO, F.; DAROS, T. A sala de aula inovadora: estratégias pedagógicas para fomentar o aprendizado ativo. 1. ed. Porto Alegre: Penso, 2018.

COLE, J.E.; WASBURN-MOSES, L.H. Going beyond "the math wars". A special educator's guide to understanding and assisting with inquiry-based teaching mathematics. Teaching Exceptional Children, V 42. N. 1, pp. 14-21, 2010.

FURTADO, Aline Fernanda. Um estudo sobre o desafio do ensino de engenharia, frente aos problemas econômicos, energéticos e a sustentabilidade. Revista Triãngulo, V 6. n.1, pp 3-21, 2013.

LARMER, J.; MERGEN-DOLLER, J.R. 7 Essential for Project-based Learning. Educacional Leadership, v. 68, n. 1, pp. 34-37, 2020.

MANRIQUE, A. L.; DIRANI, E. A. T.; CAMPOS, L. C. PBL em um curso de Engenharia Biomédica: a experiência da PUC-SP. International Conference-Problem-Based Learning and Active Learning Methodologies. Anais. São Paulo, 2010.

MAZUR, Erik. Peer Instruction: a revolução da aprendizagem ativa. Porto Alegre. Penso:2015.

MINISTÉRIO DA EDUCAÇÃO. Resoluçao n ${ }^{\circ}$ 2, de 24 de abril de 2019. Institui as Diretrizes Curriculares Nacionais do Curso de Graduação em Engenharia. Institui as Diretrizes Curriculares Nacionais do Curso de Graduação em Engenharia. Disponível em 
http://portal.mec.gov.br/index.php?option=com_docman\&view=download\&alias=112681rces002-19\&category_slug=abril-2019-pdf\&Itemid=30192. Acesso: 03 Mar 2019.

ROCHA, Martins; LEMOS, Washington de Macedo. Metodologias ativas: do que estamos falando? Base conceitual e relato de pesquisa em andamento. IX Simpósio Pedagógico e de Pesquisa em Educação. AEDB. Rio de Janeiro. Anais. Resende, 2014.

SEMESP. STHEM Brasil. Sindicato das Entidades Mantenedoras de Estabelecimentos de Ensino Superior no Estado de São Paulo, 09 out. 2019. Disponível em . Acesso em 09 ago. 2020.

SILVA, Fabiana Sena da. A noção de competência no ensino superior: o curso de pedagogia da UFPB. RBPA. v. 23, n. 2, pp. 315-320, 2007.

UNESCO. Educação para a cidadania global: preparando alunos para os desafios do século XXI. Brasília: UNESCO, 2015. Disponível em: https://unesdoc.unesco.org/ark:/48223/pf0000234311. Acesso: 09 mar. 2019.

\section{PROJECT-BASED LEARNING APPLIED TO ENGINEERING TEACHING: MOTIVATION AND MOBILIZATION SKILLS ACTIVATION}

Abstract: The new relationship between technology, production and employment has brought a challenge to educational institutions, with regard to the training of professionals worldwide. The path indicated is that of competence-oriented teaching as an answer to this challenge, since the current model based on the traditional method is not effective. This fact may be related to this teaching methodology, which makes it inefficient and demotivating for students. Active teaching methodologies and, among them, Project Based Learning, are aligned with this new reality in Engineering courses. This article seeks to understand, through a symmetrical scale assessment questionnaire, the personal perception of engineering students after applying this methodology in a discipline of the professional cycle, during an academic semester, with the participation of a company in the automotive segment. The results indicated the effectiveness of Project Based Learning, in relation to the mobilization of students' skills and motivation.

Keywords: Project-Based Learning. Active methodologies. Engineering Teaching. 